\title{
ASPECTOS GERAIS DA AVALIAÇÃO PRÉ-OPERATÓRIA DO PACIENTE IDOSO CIRÚRGICO
}

\author{
General features of the preoperative assessment of surgical elderly patient
}

Soraya VENDITES, Clineo de Melo ALMADA-FILHO, José Guilherme MINOSSI

Trabalho realizado na Disciplina de Geriatria e Gerontologia da UNIFESP - EPM, São Paulo, SP, Brasil.

DESCRITORES - Cirurgia. Avaliação préoperatória. Idoso.
RESUMO - As intervenções cirúrgicas em pacientes idosos têm se tornado cada vez mais frequentes, uma vez que esta população vem apresentando crescimento em número significativo nas últimas décadas e consequentemente expondo-se por mais tempo às morbidades de resolução cirúrgica. Apesar de um número importante de idosos apresentarem envelhecimento saudável e tolerarem o ato cirúrgico sem muitas intercorrências, sabe-se também que a morbi-motalidade que envolve a operação é mais frequente no indivíduo idoso e requer muito mais cuidado naquele com comorbidades. O presente trabalho se propõe a rever alguns aspectos que merecem ser mais cuidadosamente observados, porque envolvem modificações morfológicas e funcionais do organismo senescente, que guardam relação com o aumento da morbidade e da mortalidade em todo o período peri-operatório. A avaliação pré-operatória, portanto, deve pautar-se em exame criterioso de toda história clínica do paciente com vistas a estabelecer medidas de prevenção de eventos mórbidos, evitar iatrogenias, estabelecer critérios que alertem para um maior risco e desenvolver enfoque multidisciplinar.

\section{Correspondência: Soraya Vendites \\ e-mail: svendites@uol.com.br \\ Fonte de financiamento: não há \\ Conflito de interesses: não há \\ Recebido para publicação: 15/04/2010 \\ Aceito para publicação: 14/06/2010}

HEADINGS - Surgery. Preoperative assessment. Elderly.
ABSTRACT - The surgical interventions in elderly patients have become more frequent once this population have presented expressive increase in last decades, resulting a longer exposure to surgical resolution morbidities. Despite a large number of elder people presents a healthy aging and support the surgical act well, the morbidity and mortality that involves the surgery is more often in elderly and request more care to ones with comorbidities. The present work purpose to review some aspects that must be more carefully observed because involves morphological and functional changes from senescent organism that bring into relation with the increase of morbidity and mortality in preoperative period. Thus, the preoperative evaluation must be based on carefully clinical examination in order to prevent morbid events, iatrogenic ones, to set up risk criteria and to development a multidisciplinary focus.

\section{INTRODUÇÃO}

$\mathrm{S}$ abemos que a população mundial vem apresentando queda significativa nas taxas de fecundidade e também nas de mortalidade. Em especial a população brasileira, que já vem assistindo a um franco processo de envelhecimento, ocupará até 2025 a $6^{a}$ posição como maior população de idosos do planeta.

Rapidamente vão ocorrendo mudanças no perfil de morbidade e mortalidade que a longo prazo vão gerando incapacidade, dependência na vida diária e perda da autonomia ${ }^{61}$ Neste contexto insere-se o tratamento cirúrgico do paciente idoso, tendo o desafio de resolver ou minimizar situações patológicas, e devolvendo o indivíduo o mais breve possível para sua rotina habitual sob pena de subtrair-Ihe a funcionalidade. Além disto, a decisão de um tratamento cirúrgico, seja ele eletivo ou de urgência, engloba uma gama de aspectos relativos à geriatria, dos quais devem estar conscientes o médico cirurgião, o anestesista, o clínico assistente e toda a equipe multidisciplinar envolvida. Cabe ressaltar estimativas de que os pacientes com mais de 65 anos respondem, em média, por $65 \%$ da atividade exercida por um cirurgião geral ${ }^{40}$.

A incidência de complicações perioperatórias está diretamente 
relacionada à idade, à presença de comorbidades e à urgência da operação. Deve-se ressaltar, no entanto, que os idosos constituem um grupo heterogêneo de indivíduos e que a idade biológica, a qual traduz o estado funcional do paciente, é mais importante que a idade cronológica propriamente dita ${ }^{37}$. Uma hospitalização pode resultar em tamanha perda funcional que torna necessária liberação para um serviço de "cuidados especiais de enfermagem" no momento da alta hospitalar ${ }^{53}$.

Além de seu parecer pessoal, pautado em exame clínico criterioso, o médico precisará valer-se de alguns instrumentos que tornem a linguagem comum quando se pretende quantificar o risco daquele procedimento, ou prognosticar determinadas complicações com maior ou menor frequência. Para tal lança-se mão de vários índices existentes na literatura médica, que apresentam critérios baseados em estado físico, funcional, cardíaco ou nutricional do paciente e os utiliza como um elemento a mais na sua avaliação pré-operatória. É importante lembrar a inexistência de índices específicos para a população idosa e que é a experiência da equipe, o conhecimento da estrutura do serviço, a habilidade e o conhecimento do cirurgião - além de um bom entrosamento da equipe multidisciplinar - que determinarão o prognóstico mais próximo do resultado a que se chegará.

O objetivo deste estudo é o de avaliar os aspectos envolvidos nessa avaliação clínica pré-operatória, de uma maneira geral e também prática, revendo as publicações médicas e as recomendações utilizadas mais recentemente que influenciam a boa prática médica.

\section{Índices de risco}

A utilização de alguns índices de risco é útil em apresentar estimativa da probabilidade de eventos nefastos, embora apresentem imperfeições metodológicas quando aplicados às diversas populações que se submetem à operação. A validade e a aplicabilidade se dão somente naquela determinada população, naquele mesmo hospital e na mesma época de desenvolvimento tecnológico ${ }^{59}$.

Goldman et al. (Tabela 1 ) 22 foram os primeiros a desenvolver um índice de risco pré-operatório com preditores multifatoriais. Esses autores avaliaram 1001 pacientes consecutivos que foram submetidos à operação não cardíaca e descreveram nove variáveis associadas com o aumento do risco para complicações perioperatórias. Estabeleceram pontuação que leva à uma classificação associada à complicações fatais e não fatais.

Como já mencionado, escalas de risco cirúrgico que tenham implicações prognósticas em relação ao ato operatório têm sido estudadas e aplicadas nesta avaliação, mas nenhuma delas é específica para o indivíduo idoso. É bem conhecida a Classificação do Estado Físico utilizada pela ASA (American Society of Anesthesiologists)(Figura 1).
TABELA 1 - Índice de Goldman e colaboradores ${ }^{22}$

\begin{tabular}{|c|c|c|}
\hline \multicolumn{2}{|l|}{ Fatores de risco } & Pontuação \\
\hline \multicolumn{2}{|l|}{ Idade $>70$ anos } & 5 \\
\hline \multicolumn{2}{|l|}{ Infarto do miocárdio há menos de seis meses } & 10 \\
\hline \multicolumn{2}{|l|}{ Presença de $3^{a}$ bulha ou estase jugular } & 11 \\
\hline \multicolumn{2}{|l|}{ Estenose aórtica grave } & 3 \\
\hline \multicolumn{2}{|c|}{$\begin{array}{l}\text { Ritmo diferente do sinusal ou extra-sístoles supra-ventriculares no } \\
\text { eletrocardiograma pré-operatório }\end{array}$} & 7 \\
\hline \multicolumn{2}{|c|}{$\begin{array}{l}\text { Mais de cinco extra-sístoles ventriculares documentadas em } \\
\text { qualquer eletrocardiograma, qualquer período }\end{array}$} & 7 \\
\hline \multicolumn{2}{|c|}{$\begin{array}{l}\mathrm{PaO} 2<60 \text { ou } \mathrm{PaCO} 2>50 \mathrm{mmHg} ; \mathrm{K}<3 \text { ou } \mathrm{HCO} 3<20 \mathrm{mEq} / \mathrm{L} ; \\
\text { uréia }>50 \text { ou creatinina }>3 \mathrm{mg} / \mathrm{dL} ; \text { transaminases anormais; } \\
\text { sinais de doença crônica hepática ou o paciente acamado }\end{array}$} & 3 \\
\hline \multicolumn{2}{|c|}{ Operação envolvendo a aorta, intraperitoneal ou intratorácica } & 3 \\
\hline \multicolumn{2}{|c|}{ Operação de emergência } & 4 \\
\hline Classe & $\begin{array}{l}\text { Complicações Com } \\
\text { não fatais (\%) fat }\end{array}$ & $\begin{array}{l}\text { nplicações } \\
\text { tais (\%) }\end{array}$ \\
\hline Classe I (0-5 pontos) baixo risco & 0,2 & \\
\hline Classe II (6-12 pontos) risco intermediário & 5 & \\
\hline Classe III (13-25 pontos) risco alto & 11 & \\
\hline Classe IV ( $>26$ pontos) risco alto & 22 & \\
\hline
\end{tabular}

\begin{tabular}{|l|l|}
\hline Classe I & Normal. Paciente saudável \\
Classe II & Doença sistêmica moderada (ex, diabetes) \\
Classe III & $\begin{array}{l}\text { Doença sistêmica severa (ex, falência renal, cirrose) } \\
\text { Doença sistêmica severa que é uma constante ameaça à } \\
\text { vida (ex, ICC severa }\end{array}$ \\
Classe IV & $\begin{array}{l}\text { Paciente moribundo. Sem expectativa de sobreviver sem a } \\
\text { operação }\end{array}$ \\
Classe V & $\begin{array}{l}\text { Paciente com morte cerebral declarada do qual órgãos } \\
\text { serão removidos para doação }\end{array}$ \\
Classe VI &
\end{tabular}

FIGURA 1 - Classificação de Estado Físico da Sociedade Americana de anestesiologistas

Os estudos têm mostrado que a classificação da ASA é um preditor de morbi-mortalidade inclusive para o indivíduo idoso ${ }^{2}$. Essas escalas, no entanto, são apenas referência no contexto geral em que o médico se ampara. O resultado do tratamento é excelente a partir de uma sequência ininterrupta: correta e absoluta indicação cirúrgica, e operação, urgente ou eletiva, realizada por irrestrita necessidade. Indicação cirúrgica intempestiva não deixa de constituir o primeiro passo direcionado ao fracasso terapêutico - imagem cristalina de um péssimo risco cirúrgico ${ }^{58}$. Elevar o custo operacional com solicitações de exames sem obedecer a criterioso raciocínio clínico não faz sentido e não traz vantagem para o paciente. Nenhum exame complementar foi capaz de orientar ou assumir com absoluta certeza a contra-indicação de uma intervenção cirúrgica ${ }^{58}$.

As mudanças associadas à idade predispõem a população geriátrica a riscos únicos, provavelmente ausentes em populações mais jovens, por exemplos a insuficiência cardíaca congestiva, a doença isquêmica do coração, a broncoaspiração, o delirium, a pneumonia e infecção do trato urinário ${ }^{71}$. Além destes, pode-se identificar síndrome de fragilidade, estado comumente encontrado neste grupo, não estando proporcionalmente associada à idade mas às comorbidades. Alguns poderão não ser tão idosos e ter fragilidade, outros serão muito idosos e não a apresentarão, criando-se subgrupos nesta população. 


\section{Aspectos relevantes do exame clínico}

A alta prevalência de múltiplas comorbidades no idoso remete à necessidade de história e de exame físico completos. O tipo de procedimento, assim como a presença de vários fatores de risco podem ser identificados com uma revisão da história clínica do paciente ${ }^{29}$. O objetivo principal nesse momento é a identificação de fatores que possam ser otimizados antes da operação visando melhorar os resultados. Também ajuda a alertar para cuidados de condições clínicas que possam afetar a condução do procedimento perioperatório. Comorbidades pré-existentes como doença arterial coronariana, enfisema ou insuficiência renal, têm impacto maior no resultado da operação que a idade cronológica.

\section{Aspectos cardiovasculares}

O idoso saudável geralmente apresenta os sinais cardiovasculares sem alterações no repouso, mas quando submetido a estresse eles passam a evidenciar reserva cardiovascular deficitária.

A avaliação cardíaca completa também inclui capacidade funcional baseada na tolerância ao exercício ${ }^{16}$. Ela pode ser objetivamente quantificada em equivalentes metabólicos (METs). Uma unidade MET representa o consumo de oxigênio de um homem de $70 \mathrm{Kg}$ em repouso. Pacientes que não são capazes de realizar atividades maiores que 4 METs estão sob risco aumentado para complicações cardíacas $^{16}$. Spin e colaboradores encontraram que para cada 1 MET adquirido houve redução de $11 \%$ na mortalidade anual ${ }^{72}$. Pacientes com baixa tolerância ao exercício físico deveriam ser examinados mais além, e otimizados quando possível ${ }^{77}$.

Os maiores preditores de complicações cardíacas incluem infarto do miocárdio dentro dos seis meses precedentes, angina do peito severa, insuficiência cardíaca congestiva descompensada, doença valvar severa, síndrome coronariana aguda e arritmia ventricular.

Até bem pouco tempo era preconizada a resolução de lesão coronária antes da realização de operações de grande porte em pacientes coronarianos que apresentassem sinais e sintomas de isquemia com comprovação por exames subsidiários ${ }^{35}$. No entanto, o estudo CASS (Coronary Artery Surgery Study) mostrou que a mortalidade cardiovascular em pacientes portadores de insuficiência coronária controlada no préoperatório e com provas de isquemia normais - apesar da evidência de obstrução coronária pelo cateterismo -, era igual tanto para o grupo que realizou terapia de reperfusão miocárdica quanto para aqueles que não o fizeram antes da operação vascular ${ }^{19}$. A necessidade de intervenção pré-operatória está reservada, portanto para os casos agudos e sintomáticos ${ }^{20}$.

O uso de beta-bloqueadores no periopertório para redução do risco cardíaco foi extensivamente estudado. Metanálise realizada por McGory et al. ${ }^{42}$, incluindo ensaios clínicos randomizados, encontrou redução de risco significante estatisticamente para a mortalidade cardíaca em pacientes de risco. Auerbach et al. ${ }^{6}$ também mostraram este efeito. Os beta-bloqueadores mais utilizados para esse fim têm sido o atenolol e o bisoprolol, que foram testados nos estudos ${ }^{39,80}$. Eles são iniciados no pré-operatório e mantidos até a alta hospitalar ${ }^{32}$. Evita-se o uso desses medicamentos, ou são utilizados com cautela, nos idosos com níveis de frequência cardíaca inferiores a 55 batimentos por minuto e/ou de pressão arterial sistólica inferior a 100 $\mathrm{mmHg}^{37}$.

O uso de estatinas também está associado à maior proteção de eventos coronários pré-operatórios, conforme estudos mais recentes ${ }^{56}$.

Ainda abordando aspectos cardiovasculares, a hipertensão arterial, por exemplo, com alta prevalência na população adulta - especialmente naquela com mais de 65 anos de idade -, faz dela problema comum nos pacientes submetidos à procedimentos cirúrgicos. Estima-se que $61 \%$ dos portadores de doença isquêmica miocárdica submetidos a operações, em geral sejam hipertensos ${ }^{38}$.

A avaliação do risco cirúrgico de paciente hipertenso é um dos principais e mais comuns motivos para a solicitação de consulta pré-operatória ${ }^{78}$. O cirurgião deve conhecer algumas alterações fisiológicas a ela relacionadas.

O paciente hipertenso desenvolve resposta cardiovascular adaptativa aos períodos prolongados de elevação da pressão arterial, como hipertrofia do ventrículo esquerdo, que acarreta elevação da pressão diastólica final e déficit de relaxamento do ventrículo esquerdo (disfunção diastólica), redução da reserva coronária e disfunção endotelial ${ }^{63}$.

Rocha e Rocha ${ }^{63}$ fazem, dentre outras, as seguintes considerações: 1) pacientes com pressão arterial igual ou inferior a 179/109 mmHg, sem lesão de órgãoalvo, têm risco cirúrgico semelhante ao normotenso; 2) adiar operação, nesses casos, traz grande frustração ao paciente e à equipe cirúrgica e eleva os custos hospitalares; 3) operações eletivas em pacientes com pressão arterial superior a 180/110 mmHg devem ser adiadas, pois há risco de complicações intra e pósoperatórias; 4) toda medicação anti-hipertensiva deve ser tomada até o dia da operação e ser reinstituída no pós-operatório o mais breve possível, no sentido de se evitar hipertensão pós-operatória; 5) nas operações de urgência e também nas eletivas, o controle das oscilações de pressão arterial pelo anestesista é mais importante para se evitar complicações do que o controle de pressão arterial no pré-operatório; 6) se o risco cirúrgico da hipertensão arterial isoladamente não é significativo, o mesmo não se pode dizer quando existe lesão associada de órgãos-alvo. Nesse caso, o risco cirúrgico é aumentado de acordo com a severidade de acometimento do órgão-alvo e também da magnitude do ato cirúrgico. 
Aspectos Da Profilaxia Para Trombose Venosa Profunda (TVP) E Tromboembolismo Pulmonar (TEP)

Com relação à profilaxia para TVP e TEP, devese considerar que a detecção, especialmente de TVP, pode ser difícil, pois apenas cerca de metade dos pacientes tem quadro clínico evidente e não há método diagnóstico simples com sensibilidade e especificidade suficientes para detecção da maioria dos quadros de trombose. Além disto, a necessidade de profilaxia prolongada pode trazer inconvenientes como a possibilidade de plaquetopenia induzida por heparina, ou a dificuldade de arcar com os custos de heparinas fracionadas por tempo prolongado ${ }^{54}$.

A incidência de TVP aumenta de 1.8/1000 indivíduos com idade entre 65 a 69 anos para 3.1/1000 indivíduos entre 85 e 90 anos. É grande a incidência de TVP nos pacientes submetidos à grandes operações abdominais, neurológicas, ginecológicas e ortopédicas, podendo atingir nesta última, 30-50\% dos indivíduos ${ }^{25}$.

Considerar ajuste de dose para a função renal, especialmente para heparina de baixo peso molecular e outros agentes com eliminação renal, especialmente em idosos e pacientes com alto risco de sangramento. Em todo paciente submetido à anestesia ou à analgesia no neuroeixo, deve-se ter precaução com o uso de profilaxia com anticoagulante ${ }^{54}$.

A predisposição para a trombose intravascular entre os idosos reflete processo multifatorial de variáveis clínicas, sugerindo o envolvimento de fatores sistêmicos isolados ou combinados ${ }^{63}$.

$\mathrm{Na}$ fisiopatologia que leva à formação do êmbolo no paciente geriátrico pode-se eleger dois fatores: 1 ) estase venosa - a diminuição da velocidade circulatória venosa no idoso está intimamente relacionada com a diminuição do débito cardíaco, com as alterações da parede e do conteúdo dos vasos (calibre, elasticidade e policitemia) e com a imobilidade; 2) lesões da parede do vaso - operações abdominais extensas, por determinarem múltiplas lesões das paredes dos vasos, favorecem o aparecimento de trombos. Também deve ser evitada a utilização de veias do membro inferior para a instalação de soluções durante o procedimento cirúrgico. Admite-se mesmo contra-indicação por esta via quando houver varizes ${ }^{52}$.

Entre os fatores de risco, chamam atenção a idade, a imobilidade prolongada, a insuficiência cardíaca especialmente quando associada a outras condições, como: miocardiopatia dilatada, diminuição da fração de ejeção e fibrilação atrial, trombofilias adquiridas (destaque para a síndrome do anticorpo antifosfolipídeo), trombofilias hereditárias (deficiência de proteínas envolvidas no processo de coagulação, como a antitrombina III e proteína C.

Avaliação de risco utilizada por importante hospital de São Paulo é a seguinte ${ }^{54}$ :

1) baixo risco: operações em pacientes com menos de 40 anos, sem outros fatores de risco; operações de pequeno porte (com duração inferior a $30 \mathrm{~m}$ e sem necessidade de repouso prolongado) em pacientes com mais de 40 anos de idade; trauma menor;

2) médio risco: operação de grande porte (abdominal, urológica ou ginecológica) em pacientes com 40 a 60 anos, sem outros fatores de risco; operação abdominal e ginecológica em pacientes com menos de 40 anos tomando estrógenos;

3) alto risco: operação geral em pacientes com mais de 60 anos; operação geral em pacientes entre 40 e 60 anos com fatores de risco adicionais; operação maior em pacientes com história de TVP ou TEP pregressa ou trombofilia; grandes amputações; operações ortopédicas maiores; operações maiores em pacientes com neoplasias malignas; operações maiores em pacientes com outros estados de hipercoagulabilidade; traumas múltiplos com fraturas de pelve, quadril ou membros inferiores.

A Tabela $2^{54}$ faz referência a outros fatores de risco individuais podendo observar-se que a maioria das condições apresentadas é mais comum no indivíduo idoso. Na Tabela 3 transcreve-se a profilaxia utilizada por esse mesmo serviço ${ }^{54}$.

TABELA 2 - Fatores de risco para TEP / TVP

\begin{tabular}{ll}
\hline Fatores de alto risco & Fatores de moderado risco \\
\hline . Idade $\geq 60$ anos & . Varizes / insuficiência venosa periférica \\
\hline . Paresia / imobilidade & . Doença inflamatória intestinal \\
\hline História prévia de TEP / TVP & . História familiar de TEP/TVP \\
. ICC descompensada & . Síndrome nefrótica \\
\hline . Insuficiência respiratória & . Doença reumatológica ativa \\
\hline . Infecção grave - sepse & . Cateter venoso central \\
\hline Trombofilia conhecida & . Obesidade - IMC $>35$ \\
\hline . Doença mieloproliferativa & . Contraceptivo / TRH \\
\hline . Câncer em atividade & . Puerpério (até 4 sem) \\
\hline .QT / RT / T hormonal & . Abortamento recorrente \\
\hline
\end{tabular}

TABELA 3 - Profilaxia de TEP / TVP

\begin{tabular}{|c|c|c|}
\hline $\begin{array}{l}\text { HNF- heparina não } \\
\text { fracionada }\end{array}$ & $\begin{array}{l}\text { Heparina (liquemine): } 5000 U I \\
\text { SC a cada 8-12 horas }\end{array}$ & Eliminação hepática \\
\hline $\begin{array}{l}\text { HBPM - heparina de } \\
\text { baixo peso molecular } \\
\text { Doses: < } 3400 \text { UI e > } \\
3400 \text { UI }\end{array}$ & $\begin{array}{l}\text { Enoxaparina (Clexane) de } 20 \\
\text { mg SC } 1 x / \text { dia a } 40 \text { mg SC } \\
1 x / \text { dia } \\
\text { Nadroparina (Fraxiparine) de } \\
2850 \text { a } 5700 \text { UI SC } 1 \text { x/dia }\end{array}$ & Eliminação renal \\
\hline Varfarina & Manter INR 2 a 3 & $\begin{array}{l}\text { Metabolismo } \\
\text { hepático } \\
\text { Excreção renal }\end{array}$ \\
\hline $\begin{array}{l}\text { CPI - Compressão } \\
\text { pneumática intermitente }\end{array}$ & & Perneira P/M/G \\
\hline $\begin{array}{l}\text { Meias elásticas de } \\
\text { compressão graduada }\end{array}$ & & $\mathrm{P} / \mathrm{M} / \mathrm{G}$ \\
\hline Compressão plantar & $\begin{array}{l}\text { Alternativa à CPI em caso de } \\
\text { lesão de perna/coxa }\end{array}$ & $\begin{array}{l}\text { Botas de tamanho } \\
\text { único }\end{array}$ \\
\hline
\end{tabular}

Sabe-se que os fatores de risco relatados variam amplamente e a independência e magnitude de cada um é incerta. Falhas em reduzir os índices de eventos trombóticos podem ser resultado de fatores de risco relativamente duvidosos e associada dificuldade em reconhecer os indivíduos sob risco. ${ }^{26}$

A falta de uniformidade na decisão sobre a tromboprofilaxia após as grandes operações 
ortopédicas é um problema que atinge clínicos, ortopedistas e todos que tratam do tromboembolismo venoso. Há grande polêmica sobre como usar e o que usar na profilaxia, bem como o seu tempo, intra e extrahospitalar.

\section{Aspectos do balanço hidro-eletrolítico}

A redução da homeostase é característica do processo de envelhecimento e confere ao indivíduo idoso mais susceptibilidade ao estresse ambiental, menoradaptação às mudanças e prejuízo na recuperação da agressão ou injúria ${ }^{10}$. As alterações fisiológicas que normalmente ocorrem com o avançar da idade, relacionadas ao equilíbrio da água e dos eletrólitos, têm expressões renais anatômicas e funcionais pela perda substancial da massa renal total (de um peso de 250 a $340 \mathrm{~g}$ nos adultos jovens para 180 a $200 \mathrm{~g}$ aos 80-90 anos) $)^{44}$, à custa de sofrimento da camada cortical com hialinização da parede dos vasos e destruição da unidade funcional, mas com comprometimento funcional capaz de manter o trabalho renal qualificado em faixa tolerável na maioria das situações de vida de uma pessoa idosa. ${ }^{67}$. Há importante diminuição na taxa de filtração glomerular após a quarta década de aproximadamente $10 \mathrm{~mL} / \mathrm{min}$ por década; entretanto $30 \%$ dos indivíduos idosos normais não a exibem ${ }^{8}$. Apesar da diminuição na taxa de filtração glomerular, a creatinina sérica tende a permanecer constante devido à progressiva queda na massa muscular ${ }^{47}$.

Assim, os aspectos mais importantes do impedimento funcional dizem respeito à capacidade diminuída para produzir urina concentrada e a limitada aptidão renal para excretar água, os eletrólitos e os radicais ácidos ${ }^{67}$.

Tendo em mente todas estas constatações, é preciso entender que ao inevitável estresse do ato operatório, não se pode acrescentar a impropriedade de reposição hidreletrolítica inadequada em termos de qualidade e quantidade de solutos, de volume da solução e quantidade de medicamentos administrados, podendo as iatrogenias decorrentes romperem 0 delicado equilíbrio mantido à custa muito mais de adaptação do que de mecanismos compensadores ${ }^{8}$.

\section{Água e hormônios}

A capacidade de excreção de água é dependente de fluxo plasmático renal suficiente e da taxa de filtração glomerular, de alças de Henle e túbulos distais funcionantes e supressão da secreção de vasopressina, ou hormônio anti-diuréico (ADH). Há no indivíduo idoso diminuição na capacidade de concentrar urina e excretar água, sendo a taxa de filtração glomerular o fator mais importante dessa capacidade dilucional. $\mathrm{O} A D H$, assim como o mecanismo de sede, mantém a osmolaridade do plasma dentro de estreita faixa de 282 a 298 mOsm/Kg e este rígido controle é essencial para a função celular normal ${ }^{43}$.

A produção de $A D H$ para uma mesma variação da osmolaridade plasmática é maior nos idosos que nos jovens, fato atribuído à maior sensibilidade dos osmorreceptores naqueles pacientes ${ }^{33,70}$. Estas alterações certamente contribuem para que o idoso tenha maior tendência à hiponatremia ${ }^{67}$.

Outro importante aspecto no equilíbrio hidreletrolítico é o papel do peptídeo atrial natriurético. Ele é sintetizado e liberado no átrio em resposta a sua dilatação e seus principais efeitos são natriurese, diurese e vasodilatação e também foi demonstrado diminuir a pressão sangüínea ${ }^{44}$.

O sistema renina-angiotensina-aldosterona também tem sua atividade diminuída com a idade e há diminuição da reposta tubular à aldosterona ${ }^{44}$. Ela age no túbulo contornado distal e nos ductos coletores, acentuando a reabsorção de sódio do líquido tubular e aumentando a excreção urinária de potássio e íons de hidrogênio. Sua diminuição pelo peptídeo atrial natriurético explica a excessiva perda de sódio do idoso e a diminuída resposta da aldosterona à hipercalemia ${ }^{45}$.

\section{Monitorização da glicemia}

Existem evidências de que controle glicêmico intensivo, mantendo valores os mais próximos ao normal, reduz risco de morte assim como septicemia, insuficiência renal aguda e transfusão sanguínea ${ }^{49}$.

Preconiza-se monitoração da glicemia capilar a cada 1 a $2 \mathrm{~h}$ antes, durante e após procedimentos cirúrgicos.

Nos pacientes que utilizam estritamente insulina, iniciar com 1 a 2 U/h de insulina EV e SG5\%, 75 a 125 $\mathrm{mL} / \mathrm{h}$, com o objetivo de manter valores de glicemia entre 100 e $150 \mathrm{mg} / \mathrm{dL}$. Alternativa é dar $1 / 2$ a 2/3 da dose usual, monitorar e suplementar com doses pequenas de insulina rápida se a glicemia for superior a $200 \mathrm{mg} / \mathrm{dL}$.

Nos pacientes em uso de agentes orais, suspender a medicação no dia do procedimento e retornar depois, juntamente com a liberação da dieta. A metformina, por exemplo, deve ser suspensa e seu retorno fica condicionado à situação clínica de segurança renal e de hidratação. A introdução precoce das biguanidas pode estar associada a quadros graves de acidose lática, sendo uma das manifestações da dor abdominal. Os redutores da ação da alfaglicosidase têm de ser suspensos, pois para sua ação o paciente deve estar se alimentando. As glitazonas podem ser mantidas, já que têm efeito prolongado e a suspensão por curto espaço de tempo não trará prejuízos ao controle glicêmico. Nos pacientes tratados usualmente com insulina, deve-se prescrever $50 \%$ da dose de insulina de ação semi lenta ou lenta na manhã da operação e suplementar com doses pequenas, conforme o resultado da glicemia capilar ${ }^{49}$.

\section{A sede}

Existem muitos mecanismos hormonais a otimizar o controle da água corporal, entretanto a única maneira de repor o déficit de fluidos é aumentar sua 
oferta e isto é controlado pela sede ${ }^{79}$. Ela e a secreção de ADH são controlados pelos mesmos estímulos de aumento da osmolaridade do plasma e diminuição do volume extracelular.

Os idosos parecem ter déficit intrínseco no mecanismo da sede, resultando em diminuição de oferta de fluidos a despeito de aumentos na osmolaridade e níveis de sódio séricos.

Em indivíduos mais jovens a sede torna-se evidente quando a osmolaridade plasmática alcança valores maiores que $292 \mathrm{mOsm} / \mathrm{Kg}^{44}$, entretanto o idoso requer nível mais alto de osmolaridade antes que a sede seja experimentada. Pacientes com AVE parecem também ter prejuízos na percepção da sede apesar de depleção de volume e hiperosmolaridade. Prejuízos cognitivos e funcionais também podem dificultar o acesso à água ${ }^{70}$.

O mecanismo que leva à redução da sede nos idosos não é bem conhecido e parece estar localizado nas vias neurais mediadas por opióides, ligando os osmoreceptores ao córtex cerebral. Atividade reduzida nestas vias resulta em redução da consciência da sede ${ }^{43,48}$.

\section{Homeostase do sódio}

O sódio é o principal cátion do espaço extracelular sendo frequentes os distúrbios de sua regulação nos idosos, por todos os motivos já comentados. O idoso tem comumente mais propensão para a hiponatremia e mais raramente para a hipernatremia, sendo esta associada à maiores índices de morbi-mortalidade nesta população ${ }^{33}$.

Alguns fatores de risco para hipernatremia são ${ }^{44}: 1$ ) aumento da perda hídrica - renal, trato gastrointestinal, pele, pulmão; 2) diminuição do aporte hídrico

Alguns fatores de risco para hiponatremia são ${ }^{34,44}$ : 1) depleção de sódio; 2) aumento do aporte de água; 3) diminuída capacidade renal de diluição e excreção de água; 4) outras.

\section{Homeostase do potássio}

O potássio corporal total diminui com a idade em decorrência da diminuição da massa corporal ${ }^{34}$, no entanto, o idoso está sujeito a risco de ambas, hipercalemia e hipocalemia . A excreção de potássio diminui com a idade devido à diminuição da massa renal e reduzida responsividade tubular à aldosterona. Adicionalmente a secreção de aldosterona em resposta à hipercalemia está reduzida ${ }^{8}$. Estes efeitos tornam o idoso, principalmente o paciente idoso cirúrgico mais susceptível à hipercalemia , em geral induzida por suplementação de potássio, por medicamentos como os diuréticos poupadores, antiinflamatórios não hormonais, anti-hipertensivos (IECA, beta-bloqueadores), heparina e digoxina ${ }^{33}$. Em geral estes pacientes são assintomáticos, mas podem ser vítimas de hipercalemia franca após o trauma cirúrgico ou após receberem medicações contendo potássio principalmente se houver algum grau de insuficiência renal associado ${ }^{33}$.

A hipocalemia é outra complicação que deve ser considerada no idoso candidato à operação - principalmente nos usuários crônicos de diuréticos - e corrigida antes do ato cirúrgico por causa de sua relação com taquiarritmias graves ${ }^{67}$.

\section{Aspectos relacionados ao sistema respiratório}

O pulmão do paciente geriátrico constitui campo propício para o desenvolvimento de complicações respiratórias, dadas alterações fisiológicas que acompanham o envelhecimento, como maior rigidez e menor complacência torácica, enfisema pulmonar crônico senil, bronquite crônica, redução da vascularização, aumento da capacidade residual funcional, entre outras. Concorrem ainda para o surgimento de complicações: o grau de disfunção respiratória, a forma de abordagem, a ressecção cirúrgica e também a dor causada pela ferida operatória.

A avaliação pulmonar do paciente idoso pode constar de simples exame físico e história, ou incluir testes espirométricos e gasometria, dependendo da função respiratória e da operação proposta. Testes específicos, como espirometria e a capacidade difusional são indicados, por exemplo, para os pacientes candidatos à ressecção pulmonar e esofagectomia ${ }^{30,76}$.

Complicações pulmonares são encontradas em $25 \%$ a $50 \%$ após procedimentos cirúrgicos maiores. Algumas medidas importantes para reduzir complicações pulmonares, são elas: 1) tratamento da doença pulmonar obstrutiva crônica de modo incisivo - os pacientes devem ser otimizados tanto quanto possível; operações eletivas devem ser adiadas na presença de infecção respiratória ativa e antibióticos profiláticos devem ser usados quando clinicamente apropriados antes destas opraçõs ${ }^{77} ; 2$ ) interrupção do tabagismo - deve ser insistentemente incentivado, advertindo o paciente para os riscos; contudo há alguma controvérsia com relação ao tempo de abstinência necessário, sendo geralmente recomendadas pelo menos oito semanas antes da operação, ${ }^{9,76 ;} 3$ ) tratamento de quadros asmáticos - necessitando até com o uso de corticoesteróides, pois seu uso por períodos curtos não aumenta a incidência de infecções ${ }^{30}$.

\section{Aspectos da avaliação cognitiva}

Pode-se dizer que uma grande área de exceção no que diz respeito aos melhores resultados que se tem observado no paciente geriátrico submetido à operações é a do prejuízo cognitivo ${ }^{24,50}$. Ele coloca o paciente idoso sob risco aumentado para delirium pós-operatório ${ }^{27}$.

Dois tipos de prejuízos são observados: delirium e disfunção cognitiva pós-operatória $(P O C D)^{71}$.

\section{Delirium}

Delirium (ou estado confusional agudo) - síndrome mental aguda causada por fatores orgânicos -, é complicação comum de operações nos pacientes idosos e está associada com substancial morbidade e prolongada hospitalização ${ }^{55}$. É mais comumente visto em pacientes idosos e a disfunção cognitiva pós-operatória é condição quase exclusiva do idoso. Pode ter impacto dramático no 
bem estar do paciente, com declínio abrupto na função cognitiva podendo levar à perda da independência, depressão, isolamento social e por fim morte ${ }^{24}$.

O delirium é comumente caracterizado pela flutuação dos níveis de consciência, distúrbio do ciclo sono/vigília, e atividade psicomotora alterada. Existem numerosas condições para ele no pós-operatório, e elas incluem idade avançada, interação de drogas, dano cerebral, operação e anestesia, hipóxia, sepse, distúrbio eletrolítico, dor, privação do sono, desordens endócrinas e metabólicas, além de causas menos aparentes como depressão, abstinência alcoólica e abuso de substâncias. É frequentemente o quadro de apresentação de doenças físicas e toxicidade por fármacos. O diagnóstico precoce e o tratamento dos fatores desencadeantes podem minimizar a duração e a severidade desta desordem ${ }^{51}$.

\section{Disfunção cognitiva pós-operatória}

A disfunção cognitiva pós-opertória (POCD) é definida como "deterioração da função intelectual que se apresenta com prejuízo da memória ou da concentração" 62 . O diagnóstico de POCD frequentemente requer avaliação neuropsicológica pré e pós-cirúrgica - razão pela qual se advoga avaliação cognitiva préoperatória em todos os pacientes que se submeterão a operações eletivas. As causas da POCD permanecem obscuras, mas hipótese plausível é a "queda funcional"24.

Alguns eventos cirúrgicos podem levar à microembolia sistêmica e cerebral e tem sido postulada como sendo a caua mais provável para a disfunção cognitiva observada no seguimento de operações de bypass em coronárias $^{50}$. Microêmbolos também são observados em procedimentos ortopédicos e deve ser a causa de POCD posterior a este tipo de operação ${ }^{71}$.

O Mini Exame do Estado Mental é uma ferramenta para screening breve que permite avaliar e quantificar "ao pé do leito", o prejuízo cognitivo ${ }^{17}$ (Tabela 4). O teste mental abreviado é outra ferramenta de avaliação rápida que também pode ser utilizada ${ }^{51}$.

A avaliação completa deve incluir a observação

TABELA 4 - Indicações típicas de suporte nutricional préoperatório $^{15}$

\begin{tabular}{ll}
\hline Enteral & Parenteral \\
\hline . Disfagia severa ou obstrução esofágica & . Peritonite difusa \\
\hline . Doenças neurológicas e musculares & . Obstrução intestinal \\
\hline . Grandes queimaduras ou traumas & . Vômito ou diarréia intratável \\
\hline . Pancreatite moderada & . ́leo paralítico \\
\hline & . Pancreatite aguda grave \\
\hline & . Fístula enterocutânea com alto débito \\
& . Isquemia intestinal \\
\hline & . Síndrome do intestino curto \\
& *Obstrução intestinal por câncer \\
\hline
\end{tabular}

dos medicamentos utilizados previamente à operação e todas as drogas não essenciais devem ser retiradas. Os medicamentos com risco aumentado para prejuízo cognitivo são: anticolinérgicos, benzodiazepínicos, sedativos, antidepressivos e antiparkinsonianianos.
As habilidades cognitivas podem ser avaliadas e, mais importante, delirium ou confusão aguda deve ser detectado para que medicações de risco possam ser interrompidas ou problemas eletrolíticos agressivamente tratados.

Pesquisas nesta área serão necessárias para identificar métodos apropriados para critério diagnóstico e identificação das causas desta condição, para que a prevenção e o tratamento tornem-se uma possibilidade.

\section{Aspectos nutricionais}

Sabe-se que a desnutrição está relacionada a resultados adversos em pacientes cirúrgicos, pois afeta a imunocompetência. Os pacientes que apresentam comprometimento nutricional têm prolongado o tempo de hospitalização, o tempo de reabilitação, apresentam maior risco de infecção e pior cicatrização, estando portanto a desnutrição relacionada ao aumento da morbi-mortalidade ${ }^{64}$.

Fazer diagnóstico preciso do estado nutricional não é o principal objetivo na avaliação pré-operatória, mas é importante que se conheçam os parâmetros mais comumente utilizados nesta avaliação para que se torne familiar, ao olhar do avaliador, as condições de maior risco.

Os exames bioquímicos mais comumente utilizados para a avaliação no idoso são: albumina, transferrina, hematócrito, hemoglobina, contagem total de linfócitos, colesterol total e frações ${ }^{21,73}$. Os sinais clínicos apresentam baixa especificidade para identificação de problemas nutricionais no indivíduo idoso. Isto ocorre porque muitos sinais podem ter como causa alterações fisiológicas decorrentes do processo de envelhecimento ou ainda, a instalação de algum processo patológico não nutricional ${ }^{66}$.

Indicadores antropométricos são essenciais na avaliação nutricional geriátrica, entretanto algumas alterações que ocorrem com 0 envelhecimento podem comprometer a determinação de diagnóstico antropométrico preciso, caso cuidados específicos não sejam tomados no sentido de neutralizar ou amenizar o efeito dessas alterações sobre a avaliação 23,57 .

Equações visando estimar o peso corporal e altura em indivíduos acamados foram validadas por Chumlea et al. ${ }^{11,12}$, e são instrumentos de grande utilidade para esse fim.

Gómez Ramos et al. ${ }^{61}$ avaliaram o estado nutricional de idosos hospitalizados e validaram a correlação da Miniavaliação Nutricional (MNA) ${ }^{74}$ com vários parâmetros bioquímicos e antropométricos e concluíram que os pacientes com mais de 80 anos mostraram alto risco para desnutrição ou desnutrição moderada e firmou a MNA como um instrumento útil, confiável e sensível para identificar os idosos hospitalizados com maior risco de desnutrição.

Embora os dados sejam esparsos, algumas evidências sugerem que suplementos nutricionais dados a pacientes em seguimento por fratura de quadril 
podem melhorar os resultados e reduzir a mortalidade ${ }^{46}$. De maneira geral, tem sido consenso entre equipes multidisciplinares, que se ofereçam 25 a $35 \mathrm{Kcal} / \mathrm{Kg} / \mathrm{dia}$, com aporte protéico de 1,5 a 2,0 g de proteínas $/ \mathrm{Kg} /$ dia aos pacientes pré-cirúrgicos ${ }^{36,64}$. Nos gravemente desnutridos, esta oferta nutricional no pré ou pósoperatório poderá causar o que se chama síndrome da realimentação ou refeeding syndrome, causada por rápido deslocamento de potássio, fósforo e magnésio do meio extracelular para o intracelular, quando uma sobrecarga de glicose chega à corrente sanguínea ${ }^{14,64}$. Para evitar a síndrome da realimentação, os autores recomendam iniciar suporte nutricional com metade das necessidades nutricionais por três a cinco dias.

O uso de terapia nutricional via enteral por sete a 15 dias antes da operação diminui os custos com suporte nutricional, tendo em vista que as fórmulas enterais são de custo inferior ao das parenterais. Além disso, há diminuição dos quadros de infecção hospitalar e de mortalidade ${ }^{15,41}$. A Tabela 4 resume as indicações do suporte nutricional enteral e parenteral.

Aspectos relacionados às operações oncológicas

Dois terços de todos os tumores sólidos ocorrem em pacientes com idade superior a 65 anos e a maioria das mortes relacionadas a câncer ocorrem dentro dessa faixa etária ${ }^{1}$. Existem evidências consideráveis que esta população recebe tratamento subpadronizado quando comparada com grupos etários mais jovens $7,18,65$.

As operações em idosos para doenças cardíacas, ortopédicas, vasculares estão se tornando crescentemente aceitas. No entanto, há permanente relutância em recomendar intervenções agressivas para as condições malignas ${ }^{1}$ ainda que a ressecção cirúrgica seja até agora aceita como a única terapia curativa para os tumores sólidos ${ }^{75}$. As operações oncológicas são preteridas nos pacientes idosos devido à presumível maior morbi-mortalidade, e à relutância em recomendá-las não está relacionada com a frequência de condições debilitantes coexistentes ou prejuízo do estado funcional ${ }^{65}$. A despeito das melhorias nas técnicas anestésicas e cirúrgicas, disponibilidade de novas drogas e cuidados perioperatórios otimizados, surpreende que ainda seja comum na prática clínica excluir o idoso do benefício de tratamento cirúrgico ${ }^{3}$.

Em algumas revisões foram identificados dois grandes temas relacionados: 1) resultados a longo prazo são independentes da idade - sempre que uma opção cirúrgica for possível, deverá ser considerada e oferecida independente da idade do paciente; 2 ) mesmo procedimentos agressivos podem ser experimentados por indivíduos com mais de 70 anos $^{5,13}$. Esses achados são apenas representativos para um pequeno grupo de pacientes idosos com câncer e em muito boa forma, os quais a condução do tratamento alcançou a sala de cirurgia. No entanto, é ainda observação importante que pacientes idosos - embora expostos a um risco cirúrgico maior -, possam submeter-se à maioria dos procedimentos cirúrgicos ${ }^{4}$.

\section{Aspectos da atuação interdisciplinar}

Em geriatria e gerontologia tem sido preconizada a composição de equipes interprofissionais, ou seja, com funcionamento em colaboração, tendo como objetivo prevenir e/ou minimizar lesões e doenças que acrescentem mais sofrimento a um contexto que já é de limitações. Sendo comum o idoso apresentar múltiplas doenças, é de se esperar também que equipes multidisciplinares devam atuar. $O$ que se espera, entretanto, é que esta aja com o máximo de sincronia e entendimento para melhor resultado preventivoterapêutico.

Pacientes de idade avançada permanecem mais tempo hospitalizados, apresentam altas taxas de mortalidade, institucionalização após internação, elevada necessidade de reabilitação e de serviços de atendimento domiciliar, tudo isto associado ao declínio funcional que ocorre com a hospitalização.

Siqueira et. al. ${ }^{69}$, avaliando 0 impacto funcional da internação hospitalar de pacientes geriátricos, constataram que os idosos com maiores comprometimentos funcionais na entrada - os que apresentaram déficit cognitivo e delirium -, foram os que pioraram funcionalmente ou faleceram durante a hospitalização e as complicações hospitalares também estiveram associadas às repercussões funcionais durante a internação.

A vulnerabilidade do paciente geriátrico também favorece as complicações de causa iatrogênica. Estas ocorrem em 29 a 38\% das hospitalizações de idosos ${ }^{31}$.

Aspectos próprios da hospitalização são deletérios ao indivíduo de idade avançada, com ênfase para o paciente cirúrgico que se expõe ao uso de medicação psicoativa, a equipamentos invasivos como cateteres e sondas e ao tempo prolongado no leito.

Durante a internação, a avaliação funcional fornece dados que são importantes no prognóstico e proporcionam linguagem comum entre os profissionais de saúde ${ }^{69}$.

Alguns instrumentos de avaliação podem ser utilizados na internação, visando identificar os indivíduos mais propensos a risco de desenvolver algum prejuízo funcional.

O Índice de Katz ${ }^{31}$ para a avaliação das atividades de vida diária (AVDs) tem sido empregado como método de fácil aplicação em diversos estudos e possibilita imediata compreensão do estado funcional/ cognitivo do paciente em questão.

Uma equipe organizada no sentido de perceber o paciente envelhecido deve atuar desde o primeiro instante de sua entrada no hospital, evitando dificuldades na comunicação e no entendimento dos procedimentos propostos.

A equipe deve ainda direcionar suas ações para a identificação e a modificação dos fatores relacionados à experiência dolorosa, conduzindo suas metas para a 
melhora das funções físicas, psicológicas, emocionais e socioculturais visando assim, corresponder às expectativas do paciente e de sua família ${ }^{68}$.

\section{CONCLUSÃO}

A população geriátrica tem crescido em número e complexidade e representará crescente aumento e diversidade na prática médica. Limitações fisiológicas e vulnerabilidades específicas devem gerar compreensão e planejamento por parte da equipe médica ${ }^{71}$.

Ainda que uma diversidade de exames possa ser solicitada para a população idosa que necessita de tratamento cirúrgico, é incerto se estes testes levariam à melhora dos resultados. A avaliação global deve incluir a verificação fisiológica, cognitiva, nutricional e funcional, mas sem dúvida, a história e o exame físico são as mais importantes ferramentas do exame pré-operatório. Atenção particular deve ser dada às comorbidades, fatores de risco cardíaco, fatores de risco para tromboembolismo, história de perda de peso e história medicamentosa. Exames complementares, se criteriosamente justificados, poderão acrescentar nesta avaliação. Alguns aspectos merecem ser mais cuidadosamente observados, porque envolvem modificações morfológicas e funcionais do organismo senescente, que guardam relação com o aumento da morbidade e mortalidade em todo o período perioperatório.

Déficits cognitivos e funcionais podem não ser passíveis de correção, mas seu reconhecimento no estado pré-operatório permite maior avaliação do risco e planejamento cirúrgico.

Índices de avaliação simples podem ser valiosos instrumentos para o reconhecimento dos pacientes mais propensos à complicações e sua utilização deve ser feita por todos os membros da equipe, promovendo linguagem comum entre profissionais e sincronismo de ações preventivas e terapêuticas.

Deve, portanto, a avaliação pré-operatória pautar-se em exame criterioso de toda a história clínica do paciente com vistas a estabelecer medidas de prevenção de eventos mórbidos, evitando iatrogenias e estabelecendo critérios que alertem para um maior risco, além de desenvolver enfoque multidisciplinar.

\section{REFERÊNCIAS}

1. Alexander HR, Turnbull $A D$, Salomone J et al. Upper abdominal cancer surgery in the very elderly. J Surg Oncol 1991; 47: 82-86.

2. American Society of Anesthesiologists. ASA Physical Status Classification System. Available at: www.asahhq.org/clinical/ physicalstatus.htm. Accessed oct 182006.

3. Audisio RA, Bozzetti F, GennariR et al. The surgical management of elderly cancer patients: recommendations of the SIOG surgical task force. Eur J Cancer 2004; 40: 926-38.

4. Audisio RA, Ramesh $H$, Longo $W E$, Zbar AP, Pope D. Preoperative assessment of surgical risk in oncogeriatric patients. The Oncologist 2005; 10: 262-68.
5. Audisio RA, Veronesi $P$, Ferrario $L$ et al. Elective surgery in gastrintestinal tumours in the aged. Ann Oncol 1997; 8: 317-27.

6. Auerbach $A D$, Goldman L. Betablockers and reduction of cardiac events in noncardiac surgery: Scientific review. JAMA 2002; 287: 1435-44.

7. 7.Ballester OF, Moscinsky L, Spiers A et al. Non-hodgkin's lymphoma in older person: a review. J Am Geriatr Soc 1993: 41: 1245-54.

8. Beck LH. The aging kidney. Defending a delicate balance of fluid and electrolytes. Geriatrics 2000; 55(4): 26-32.

9. Brooks-Brunn JA. Predictors of postoperative pulmonary complications following abdominal surgery. Chest 1997; 111: 564-71.

10. Brown WW, Davis BB, Spry LA, Wongsurawat N Malone JD, Domoto DT. Aging and the kidney. Arch Int Med 1986; 146(9): 1790-6.

11. Chumlea WC, Guo S, Roche AF, Steinbaugh ML. Prediction of body weight for the nonambulatory elderly from anthropometry. J Am Diet Assoc 1988; 88:564-8.

12. Chumlea WC, Roche AF, Steinbaugh ML. Estimating stature from knee height for persons 60 to 90 years of age. J Am Geriatr Soc 1985; 33:116-20.

13. Colorectal cancer collaborative group. Surgery for colorectal cancer in the elderly patients: a systematic review. Lancet 2000; 356:968-74.

14. Crook MA, Hally V, Panteli JV. The importance of the refeeding syndrome. Nutrition 2001; 17:632-7.

15. Duarte AC, Castellani FR. Semiologia Nutricional, RJ:Axcel, 2002: 17-57; 59-64.

16. Eagle $K A$, Brundage $B H$, Chaitman $B R$, et al. Guideline for perioperative cardiovascular evaluation for noncardiac surgery. Report of the American College of Cardiology/ American Heart Association Tack Force on Practice Guidelines. Circulation 1996; 93(6): 1278-1317.

17. Folstein MF, Folstein SE, McHugh PR. Mini-Mental State; A practical method for grading the cognitive state of patients for the clinician. J Psychiatr Res 1975; (12): 189-198.

18. Fonseca LAM. Mortalidade por cancer no estado de São Paulo: perfil atual, distribuição geográfica e tendências temporais. Tese de doutorado apresentada na USP. Fac de S Pública 1996.

19. Foster ED, Davis KB, Carpenter JA et al. Risk of noncardiac operation in patients with defined coronary disease : the coronary artery surgery study (CASS) registry experience. Ann Thorac Surg 1986; 41: 42-50.

20. Francisco YA, Póvoa R. Avaliação clínica para as cirurgias não cardíacas na doença coronária aguda. Em: Avaliação clínica préoperatória: risco cirúrgico/ Rui Povoa- G. Koogan 2006; 95:45-9.

21. Gariballa E, Sinclair AJ. Nutrition, aging and ill health. Br J Nutr 1998; 80:7-23.

22. Goldman L, Caldera DL, Nussbaum SR et al. Multifactorial index of cardiac risk in noncardiac surgical procedures. N Engl J Med 1977; 297(16): 845-50.

23. Goodman-Gruen D, Barret-Connor E. Sex differences in measures of body fat and body fat distribution in the elderly. Am J Epidemiol 1996; 143(9):898-906.

24. Greiner PA, Snowdon DA, Schimitt FA. The loss of independence in activities of daily living: The role of normal cognitive function in elderly nuns. Am J Public Health 1996; 86: 62-6.

25. Hagemeyer V, Buksman S, Vilela AL. Profilaxia do tromboembolismo venoso em cirurgias ortopédicas de idosos. R. Into, Rio de Janeiro, v. 2, n. 2, p. 1-60, maio/ago. 2004.

26. Heit JA, Silverstein MD, Mohr DN, Petterson TM, O' Fallon WM, Melton LJ. Risk factors for deep vein thrombosis and pulmonary embolism: a population-based case-control study. Arch Inter Med; 2000; 160:809-15.

27.Inouye SK. Predisposing and precipitating factors for delirium in hospitalized older patients. Dement Geriatr Cogn Disord 1999; 10: 393-400.

28. Johnson AG, Crawford GA, Kelly $D$ et al. Arginine vasopressin and osmolality in the elderly. J Am Geriatr Soc 1994; 42: 399-404.

29. Johnson JC. Princípios cirúrgicos no envelhecimento .Reichel's care of the elderly: clinical aspects of aging. Lippincott Willians\&Willians,1999.

30. Kabalin CS, Yarnold PR, Grammer LC. Low complication rate of corticosteroid treated asthmatics undergoing surgical procedures. Arch Intern Med 1995; 155: 1379-84. 
31. Katz, S et al. Studies of illness in the aged - the Index of ADL: a standardized measure of biological and psychosocial function. JAMA 1963. 185(2): 94-9.

32. King MS. Preoperative evaluation. Am Fam Physician 2000; 62:387-96.

33. Luckey $A E$, Parsa CJ. Fluid and electrolytes in the aged. Arch Surg 2003; 138: 1055-60.

34. Lye M. Eletrolyte disorders in the elderly. Clinics in endocrinology and metabolism 1984; 13(2): 377-98.

35. Macfalls EO, Ward HB, Moritz TE et al. Coronary artery revascularization after before elective major vascular surgery. $\mathrm{N}$ Engl J Med 2004; 351: 2795-804.

36. Mac Fie J, Woodcock NP, Palmer Md et al. Oral dietary supplements in pre and postoperative surgical patients: a prospective and randomizes clinical trial. Nutrition 2000; 16:723-28.

37. MaKdisse M, Oliveira A. Avaliação do paciente idoso e muito idoso. Avaliação clínica pré-operatória: risco cirúrgico/ Rui Povoa- Rio de Janeiro: G koogan, 2006, 158-63.

38. Mangano DT, Browner WS, Hollenberg M, et al. Association of perioperative myocardial ischemia with cardiac morbidity and mortality in men undergoing noncardiac surgery. $\mathrm{N}$ Engl J Med 1990; 323:1781.

39. Mangano DT, Layug E,Wallace A, Tateo I. Effect of atenolol on mortality and cardiovascular morbidity after noncardiac surgery. $\mathrm{N}$ Engl J Med 1996; 335: 1713-20.

40. Manku K, Bacchetti $P$, Leung JM.Prognostic significance of postoperative in-hospital complications in elderly patients. Long term survival. Anesth Analg 2003; 96: 583-9.

41. Mcclave S, Snider H, Spain D. Preoperative issues in clinical nutririon chest 1999; 115:64S-70S.

42. McGory ML, Maggard MA, Ko CY. A meta-analysis of perioperative beta blockade: what is the actual risk reduction? Surgery 2005; 138(2): 171-9.

43. McKenna K, Thompson C. Osmoregulation in clinical disorders of thirst appreciation. Clinical Endocrinology 1998; 49: 139-52.

44. Miller M. Fluid and electrolyte homeostasis in the elderly: physiological changes of ageing and clinical consequences. Baillieres Clinical Endocrinology and Metabolism 1997; 11(2): 367-87.

45. Miller PD, Krebs RA, Neal BJ, McIntyre DO. Hypodipsia in geriatric patients. Am J Med 1982; 73: 354-6.

46. Milne AC, Potter J, Avenell A. Protein and energy supplementation in elderly people at risk from malnutrition. Cochran Database Syst Rev 2995 Apr 18;(2): CD003288.

47. Mimran A, Ribstein J, Jover B. Aging and sodium homeostasis. Kidney International Supplement 1992; 37: s107-13.

48. Mulkerrin E, Epstein FH, Clark BA. Aldosterone responses to hyperkalemia in healthy elderly humans. J Am Soc Nephrol 1995; 6:1459-62.

49. Nasri F, de Sá JR. Diabetes Mellitus. Em: Guias de Medicina Ambulatorial e Hospitalar - UNIFESP - EPM. Manole 2005. 7: 77-94.

50. Newman MF, Kirchner JL, Phillips-Bute B et al. Longitudinal assessment of neurocognitive dysfunction after coronary artery bypass surgery. N Engl J Med 2001; 344: 395-402.

51. Ni Chonchubhair A, Valacio R, Kelly J. O'Keeffe S. Use of the abbreviated mental test to detect postoperative delirium in elderly people. British Journal of Anaesthesia 1995; 75: 481-482

52. Nicoletti RL. Anestesia no paciente geriátrico. Rev Bras Anest 1981 31(2): 147-55

53. Nierman DM, Schechter CB, Cannon LM, Meier DE. Outcome prediction model for very elderly critically ill patients. Crit Care Med 2001; 29(10): 1853-59.

54. Normatização de Condutas do Hospital Sírio Libanês - Grupo Profilaxia TVP/TEP em internados - março, 2006.

55. O' Keeffe ST, Ni Chonchubhair A. Postoperative delirium in the elderly. British Journal of Anaesthesia 1994; 73: 673-687.

56. O' Neil-Callahan K, Katsimaglis G, Tepper MR et al. Statins decrease perioperative cardiac complications in patients undergoing noncardiac vascular surgery. The statins for risk reduction in surgery (StaRRS) study. J Am Coll Cardiol 2005; 45: 336-42.
57. Perissinotto E, Pisent C, Sergi G, Grigoletto F, Enzi G. Anthropometric measurements in the elderly: Age and gender differences. Br J Nutr 2002; 87:177-86.

58. Pimenta RG. Risco cirúrgico no paciente idoso. Cirurgia Geriátrica -MEDSI Ed Médica e Científica -1998, 315-26.

59. Póvoa R, Souza D. O papel do consultor e análise crítica dos escores de risco. Avaliação clínica pré-operatória- Risco cirúrgico. Guanabara Koogan, 2006.

60. Ramos LR. A mudança de paradigma na saúde e o conceito de capacidade funcional. Em: Guias de Medicina Ambulatorial e Hospitalar - UNIFESP-EPM - Geriatria e Gerontologia 2005.

61. Ramos MJG, Valverde FMG, Álvarez CS. Estúdio del estado nutricional em la población anciana hospitalizada. Nutr Hosp. 2005; 20(4):286-92

62. Rasmussen LS Larssen K, Houx et al. The assessment of postoperative cognitive function. Acta Anaesthesiol Scand 2001; 45: 275-89.

63. Rocha JC, Rocha AT. Abordagem pré-operatória do paciente hipertenso: riscos orientações. Rev. Soc Cardiol Estado de São Paulo, mai/jun 2000, vol 10, $\mathrm{n}^{\circ} 3$.

64. Salvino RM, Dechicco RS, Seidner DL. Perioperative nutririon support: who and how. Clev Clin J Med 2004; 71: 345-51.

65. Samet J, Hunt WC, Key C et al. Choice of cancer therapy varies with age of patient. JAMA 1986; 255: 3385-90.

66. Sampaio LR. Avaliação nutricional e envelhecimento. Rev Nutr 2004; 17(4): 507-14.

67. Santos Jr. JCM. O paciente cirúrgico idoso. Rev bras Coloproct 2003; 23(4): 305-16.

68. Silva A, Reis ALB, Cordeiro RC, Lima SCCR. Reabilitação do paciente com dor persistente. Em: Guias de Medicina Ambulatorial e Hospitalar- UNIFESP-EPM 2005. Manole (18): 229-42.

69. Siqueira $A B$, Cordeiro RC, Perracini MR, Ramos LR. Impacto funcional da internação hospitalar de pacientes idosos. Rev Saúde Pública 2004. 38(5):687-94.

70. Snyder NA, Feigal DW, Arieff AI. Hypernatremia in elderly patients. A heterogeneous morbid and iatrogenic entity. Ann Intern Med 1987; 107(3): 309-19.

71. Souders JE, Rooke GA. Perioperative care for geriatric patients. Annals of Long-Term Care 2005; 13(6): 17-29.

72. Spin JM, Prakash M, Froelicher VF, et al. The prognostic value of exercise testing in elderly men. Am J Med 2002; 112(6): 453-59.

73. Sullivan DH, Sun S, Walls RC. Protein-energy undernutrition among elderly hospitalized patients: A prospective study. JAMA 1999; 281: 2013-9.

74. Vellas B, Garry Ph J, Guigoz Y: Mini Nutritional Assessment (MNA). Research and practice in the elderly. Nestlé Nutrition Workshop Series. Basel. Karger, 1999.

75. Wallach $C B$, Kurt RC. Gastrointestinal cancer in the elderly. Gatroenterol Clin North Am 1990; 19: 419-32.

76. Warner MA, Offord KP, Warner ME et al. Role of preoperative cessation of smoking and other factors in postoperative pulmonary complications: a blinded prospective study of coronary artery bypass patients. Mayo Cin Proc 1989; 64: 609-16.

77. Willians SL, Jones PB, Pofahl WE. Preoperative management of older patient- A surgeon's perspective: Part II. Clinical Geriatrics 2006;14: 7.

78. Wolfsthal SD. Is blood pressure control necessary before surgery? Med Clin North Am 1993; 77(2): 349-63.

79. Yatagai T, Kusaka I, Nakamura T et al. Close association of severe hyponatremia with exaggerated release of arginine vasopressin in elderly subjects with secondary adrenal insufficiency. Eur J Endocrinol 2003; 148: 221-6.

80.Zaugg $M$, Tagliente $T$, Lucchinetti $M$ et al. Beneficial effects from beta-adrenergic blockade in elderly patients undergoing noncardiac surgery. Anesthesiology 1999; 91: 1674-86. 